\title{
Intercom Device
}

National Cancer Institute

\section{Source}

National Cancer Institute. Intercom Device. NCI Thesaurus. Code C50006.

A communications device designed for use between rooms of a building. 